\title{
A simulation study on the effect of size gold nanoparticles on broadband light absorption in dye-sensitised solar cells
}

\author{
Karwan Wasman Qadir \\ Computation Nanotechnology Research Lab (CNRL), Department of Physics, \\ College of Education, Salahaddin University-Erbil, Erbil 44002, Kurdistan Region, Iraq. \\ e-mail: karwan.qadir@su.edu.krd
}

Received 16 September 2020; accepted 18 January 2021

\begin{abstract}
Plasmon-assisted energy conversion in dye-sensitised solar cells (DSSCs) has been achieved by applying gold (Au) nanoparticles (NPs) inside Titania $\left(\mathrm{TiO}_{2}\right)$ photoanodes. Gold nanoparticles (GNPs) were introduced into DSSCs to further enhance their power conversion efficiency (PCE). In this research work, an effort has been made to enhance the optical absorption and improve the performance of DSSCs. By utilising finite-difference time-domain (FDTD) software, GNPs with radii of 15, 25, 35, 45, 55, 65, 75 and 85 nm were produced and introduced into the $\mathrm{TiO}_{2}$ photoanode. The optimum radius for the optical absorption enhancement was found to be $85 \mathrm{~nm}$ because the effect of plasmon coupling is more significant for metal nanoparticle sizes $>60 \mathrm{~nm}$. The effect of various sizes of GNPs on light scattering has also been presented in this study. Moreover, the investigation has focused on the role of the incident angle of a light source on the absorption in $\mathrm{TiO}_{2}$ films. It was found that the optimum incident angle for the enhancement of broadband optical absorption in the wavelength range of $450-800$ $\mathrm{nm}$ is $70^{\circ}$.
\end{abstract}

Keywords: Plasmonic effect; finite-difference time-domain (FDTD) Simulations; gold (Au) nanoparticles (NPs); dye-Sensitised solar cells (DSSCs); titania $\left(\mathrm{TiO}_{2}\right)$.

PACS: 71.45.Gm; 61.46.+w; 02.70.-c; 88.40.H-; 77.84.Cg.

DOI: https://doi.org/10.31349/RevMexFis.67.509

\section{Introduction}

One of the most important events of 1991 was the report on a new low-cost chemical solar cell by the successful combination of nanostructured electrodes and efficient chargeinjecting dye technology, known as dye-sensitised solar cell (DSSC) or Grätzel cell, which belongs to the third generation of efficient and inexpensive photovoltaic cells [1]. Besides Perovskite and tandem solar cells, DSSCs are considered among photovoltaic (PV) devices with the most potential. This led to remarkably increased interest in DSSCs as an alternative technology for the production of future renewable energy because of their high solar power conversion efficiencies (PCE) [2]. DSSC consists of a mesoporous (Titania) $\mathrm{TiO}_{2}$ layer adsorbing a monolayer of dye molecules, an electrolyte based redox system for charge transport, and counter electrodes to collect photogenerated carriers [3], which has high power-conversion efficiency [4]. To optimise the performance of DSSCs, research universities and institutes are now working on $\mathrm{TiO}_{2}$ photoanodes fabrication with different forms of nanostructure morphologies [5-7]. Because of their advantages, which include abundantly available inexpensive materials and potentially lower costs of fabrication, DSSCs offer a promising alternative to thin-film silicon solar cells [8]. The PCEs of existing DSSC photovoltaic devicesup to this time at their development stage-reach around 15 percent, which exceeds the efficiency of conventional amorphous silicon solar cells [9]. However, this efficiency level is still far below the efficiencies offered by crystalline silicon solar cells, Perovskite solar cells, and tandem thin-film solar cell technologies $[10,11]$.

Over the two decades, since Grätzel introduced mesoporous $\mathrm{TiO}_{2}$ nanoparticle films as photoanodes in DSSCs, much of the research efforts have focused on the design of photoanodes and counter/reference electrodes, in addition to dyes and electrolytes, to achieve higher efficiencies of DSSCs [12]. Among these, the photoanode contribution plays a vital role in improving the performance of DSSCs [13]. However, the investment of maximum conversion efficiency is not easy to achieve. The loss in efficiency is because of insufficient absorption and an internal quantum efficiency smaller than unity at long wavelengths ( $>350 \mathrm{~nm}$ ) of visible light $[14,15]$. The intensity and amount of trapped light are directly proportional to the PCE, which means that a DSSC photovoltaic device's efficiency can be further boosted by introducing light trapping management [16-18]. Generally, the light-harvesting capacity of a DSSC photovoltaic device is determined by the amount of dye adsorption via the photoanode surface and the spectral response of the dye molecules [12]. Enhancing the visible light absorption is one of the approaches to increase the PCE of DSSCs. Another convenient way is to optimize the $\mathrm{TiO}_{2}$ thin layer's thickness for lightharvesting because the number of dye molecules adsorbed on the $\mathrm{TiO}_{2}$ layer is increased. However, the PCE of DSSCs is a function of the photovoltaic cell length, implying that this approach may reduce the efficiency because it assists the recombination rate of photoelectron-hole pairs to travel all the increased distance to reach the collecting electrode [19]. 
Several approaches are available to increase the PCE of the DSSCs further, including external coatings [20,21], carrier collection, photonic structures [22-24], and diffractive layer $[25,26]$. However, such enhancements might come at a cost, negatively affecting other electrical properties, such as separate pairs of charge carriers, and, consequently, reducing the PCE [27].

The nanophotonic technology for light-harvesting management inside a photovoltaic device has been suggested as another approach to achieve high PCEs [19]. The applications of modern surface plasmon in photovoltaic technology are not recent, having possibly been first reported in 1998 by Stuart et al, [27]. Thus, surface plasmonics has become a global target for photonic development studies. It was found that by increasing the concentration of metallic nanoparticles, the photocurrent (electrical properties) of the photovoltaic cell device was improved 20 times at the long-wavelength $(800 \mathrm{~nm})$. So far, using metallic nanostructures for surface plasmonic effect has been one of the best ways to enhance light harvesting and PCE of DSSCs [28]. The plasmonic phenomenon in nanomaterials could help harvest more light from sub-wavelength antennas or generate plasmon polaritons from scattering or incident light by trapping light energy on metal NPs in a constant thickness of the $\mathrm{TiO}_{2}$ thin layer $[29,30]$. As a result, the PCE would be improved because this leads to an increase in the overall short circuit current density $\left(\mathbf{J}_{s c}\right)$ in the solar cells [27]. In recent years, the concept of plasmon resonance has been introduced to the DSSCs using noble metal nanoparticles such as gold $(\mathrm{Au})$ or silver $(\mathrm{Ag})$ because of their low reactivity. Plasmon resonance is one of the approaches adopted for further improvement of PCE of DSSCs. The light-harvesting efficiency is substantially enhanced with the localised surface plasmon resonance (LSPR) phenomena of metal nanoparticles [30,31]. The phenomenon of LSPR refers to the resonance between the oscillation of free electrons and the electromagnetic light field. The LSPR amplifies the electromagnetic light field near the metal nanoparticles and resonant oscillations of electronic clouds [27,32]. This improves the plasmonic effect via extending absorption and harvest of visible light by dye sensitisers in DSSCs [16,32].

The introduction of metallic nanoparticles (NPs) in appropriate places inside the active layer to confine visible light could provide superior performance due to improved absorption in the organic semiconductor film and enhance photocurrent generation via the effect of LSPR [1,33]. Metal NPs with sharp edges are highly desired due to the high electronic conductivity and their applications as interconnections in the bottom-up self-assembly approach towards future nanodevices. Moreover, the branched structure of Metal NPs such as star-shaped enhance the electric field around the nanoparticles and have multiple plasmon resonances resulting in polarization-dependent scattering with multiple spectral peaks and strong dielectric sensitivity into a single structure $[34,35]$.
For these potential features of the metal nano-star particles, in this research, different star-shaped GNPs were prepared and applied to $\mathrm{TiO}_{2}$ (possibly for the first time) to improve its optical absorption using FDTD software as described in a previous article by [29]. Multiple sizes of GNPs were applied to study the multiple plasmon-enhanced effects in the structure of the $\mathrm{TiO}_{2}$ layer. The effects of the size of GNPs on the scattering of light were investigated systematically. We have also studied the correlation between the light source incident angle and the optical absorption characteristics of the $\mathrm{TiO}_{2}$ thin layer to obtain an optimum angle for enhancing the photovoltaic performance of DSSCs. Clearly, more extensive measurements on the fully packaged DSSC (including electrodes) are required in future work.

\section{Result and discussion}

The FDTD (Yee's FDTD algorithm) technique was chosen from among the various available methods to report our results. This modeling technique is applied to solve and represent Maxwell's equations over a grid-based domain in the differential form. Given that the FDTD simulation calculates the magnetic $(h)$ and electric (e) fields everywhere as they evolve in time in the computational domain, it is straightforward to simulate and calculate the propagation of electromagnetic light field by using this model [36]. In the simulation technique, the results of $\mathrm{TiO}_{2}$ and Au optical constants were taken from the reports of [37] and [38], individually. We chose three GNPs embedded in $\mathrm{TiO}_{2}$ thin layer films using the Lumerical FDTD simulation technique. The mesoporous $\mathrm{TiO} 2$ active layer thickness was used as $100 \mathrm{~nm}$, depending on Shanmugam's experiment report [39]. Also, choosing this specific thickness of the $\mathrm{TiO}_{2}$ layer in this simulated study is to decrease the simulation time and space of the PC drive.

The monitors in the simulation were located at proper places to precisely calculate the absorbance $(A)$, reflectance $(R)$, and transmittance $(T)$. In the $z$-axis direction, perfectly matched layer (PML) absorbing boundary conditions were used on the upper and lower boundaries, while periodic boundary conditions (PBC) were used on both sides for the $x$ - and $y$-axis. For the structure's dimensions under simulation, we selected a length of $0.1 \mu \mathrm{m}$, a width of $0.1 \mu \mathrm{m}$, and a height of $1 \mu \mathrm{m}$ for each shape. The Lumerical FDTD Solution was performed over the visible light region in the solar spectrum. This FDTD simulation software was used to calculate the absorption rate as a function of the visible wavelength for a flat spectrum. This was done automatically by means of continuous wave $(\mathrm{CW})$ normalisation in FDTD, and the visible sunlight was modeled with a source of a plane wave. The calculation of power absorption was performed by solving Maxwell's equations, i.e., the absorbed power $L(r \rightarrow \lambda)$ per unit volume at each wavelength. In this study, we theoretically investigated the effect of introducing metallic $\mathrm{Ag}$ nanoparticles in thin-film $\mathrm{CuPc}$ on their optical absorption efficiency. 

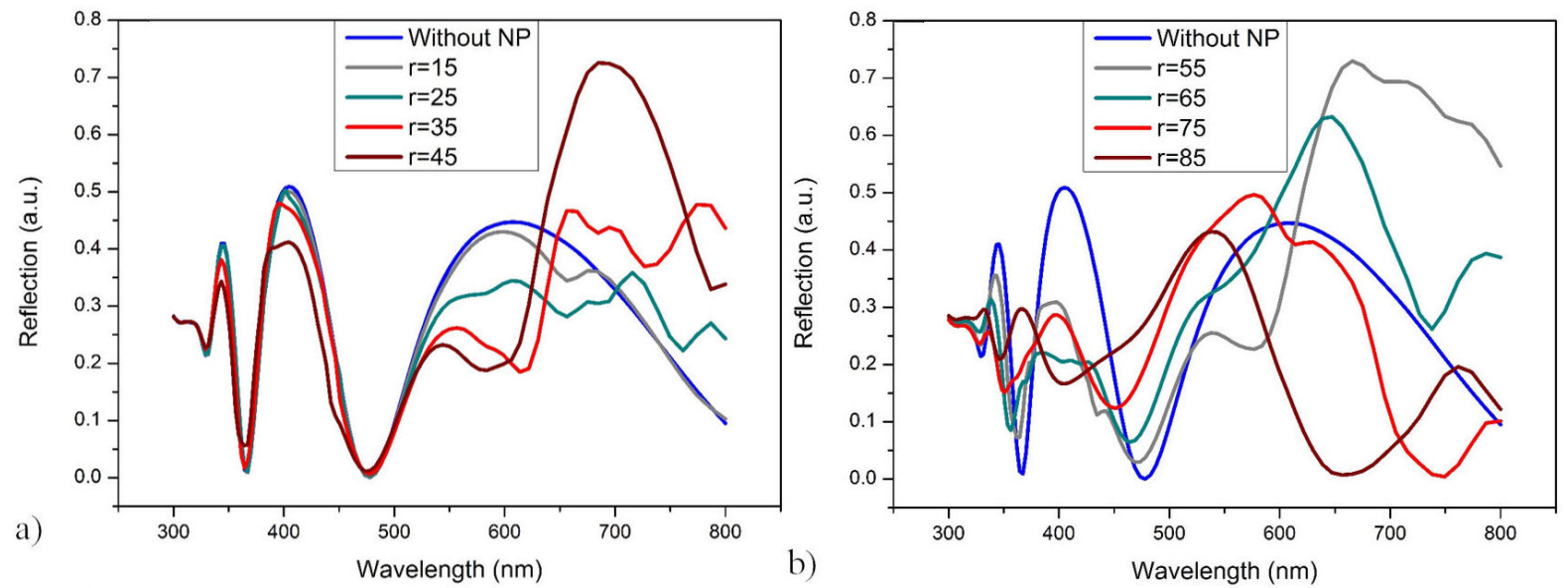

FIGURE 1. Reflection spectra of $\mathrm{TiO}_{2}$ layer with different size of Au nanoparticles.
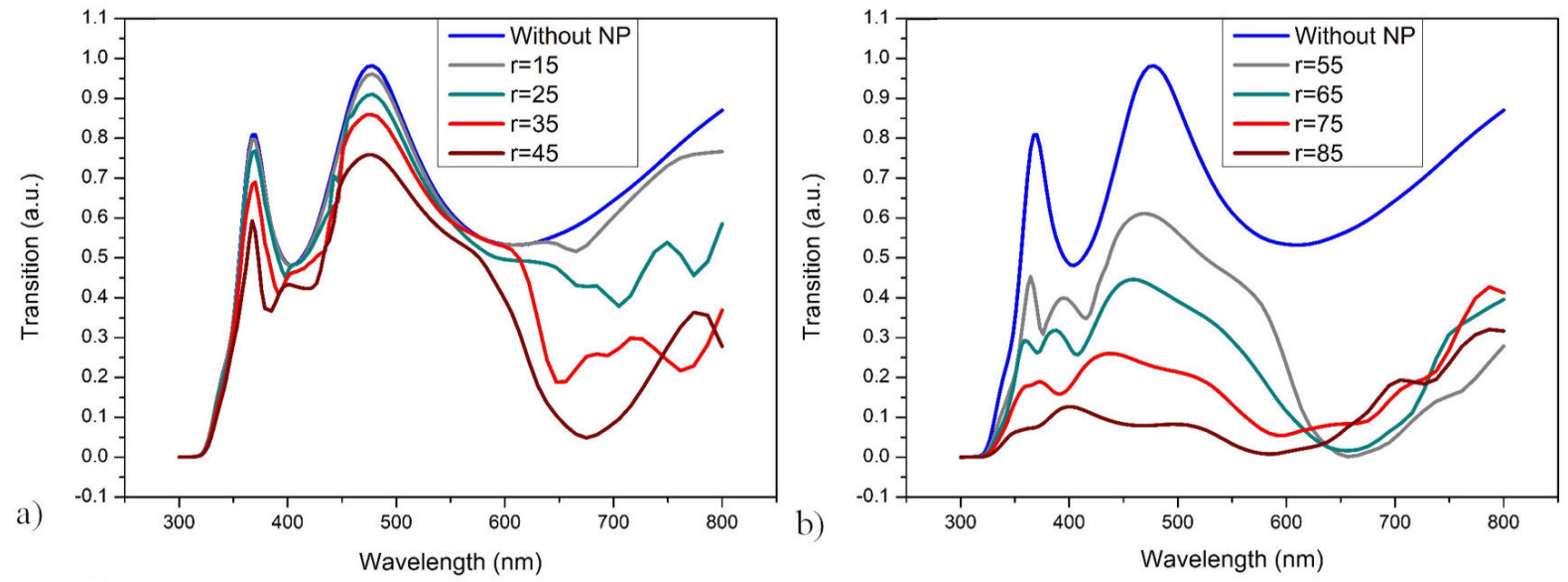

FIGURE 2. Transition spectra of $\mathrm{TiO}_{2}$ layer with different size of Au nanoparticles.

The combinations of NP shapes in the absorber layer were studied to explore the effect of nanostructures on the absorption efficiency of the $\mathrm{TiO}_{2}$ layer. Moreover, different sizes of NPs were investigated to exploit the ultimate efficiency potential in this simulation. The optical properties of $\mathrm{TiO}_{2}$ and star-shaped $\mathrm{GNP} @ \mathrm{TiO}_{2}$ thin layer in the visible spectral range of 300 to $800 \mathrm{~nm}$ were then studied.

Figure 1 shows the effect of the size of GNPs on the reflection spectra of embedded GNP@ $\mathrm{TiO}_{2}$ thin films with star shapes. Here, $r$ represents the radius of the GNPs. It is clear from the figure that NPs of different sizes have different effects on the $\mathrm{TiO}_{2}$ reflection spectra.

Similarly, it is evident from Fig. 2 that GNPs of different sizes have different effects on the $\mathrm{TiO}_{2}$ thin film transition spectra. Furthermore, the absorption spectra of GNP@ $\mathrm{TiO}_{2}$ thin films are significantly enhanced compared to those of pristine $\mathrm{TiO}_{2}$ thin films, as shown in Fig. 3. This is clearly noticeable in the visible wavelength range of 350 to $700 \mathrm{~nm}$. The results are close and comparable to the nano-star absorption spectrum of a known experimental reference [35]. The wide range and the multiple spectral peaks are because the gold nano-star particles have two plasmon resonance modes. One of them being localized at the range of $375 \mathrm{~nm}$ to 525 $\mathrm{nm}$ in the visible spectrum due to central core dipolar resonance of the GNPs. On the other hand, broad absorption spectra of star-shaped GNPs between 550 to $775 \mathrm{~nm}$ due to localized plasmon phenomenon at the tips and edge of GNPs. Rodriguez et al. suggested that the number of branches is of minor importance for determining the red-shifted band's spectral position $[34,40]$.

In this simulation study, the effect of the size of NPs on the amount of scattered light was investigated with various sizes of GNPs. As shown in Fig. 4, the different sizes of GNPs have different scattering range of the visible spectrum. Gold nanoparticles with a radius of $85 \mathrm{~nm}$ were seen to have a broader and maximum scattering spectrum compared to the other sizes. The different sizes of GNPs have different absorption spectra in the visible wavelength range of $300-800 \mathrm{~nm}$, as shown in Fig. 5. This investigation re- 

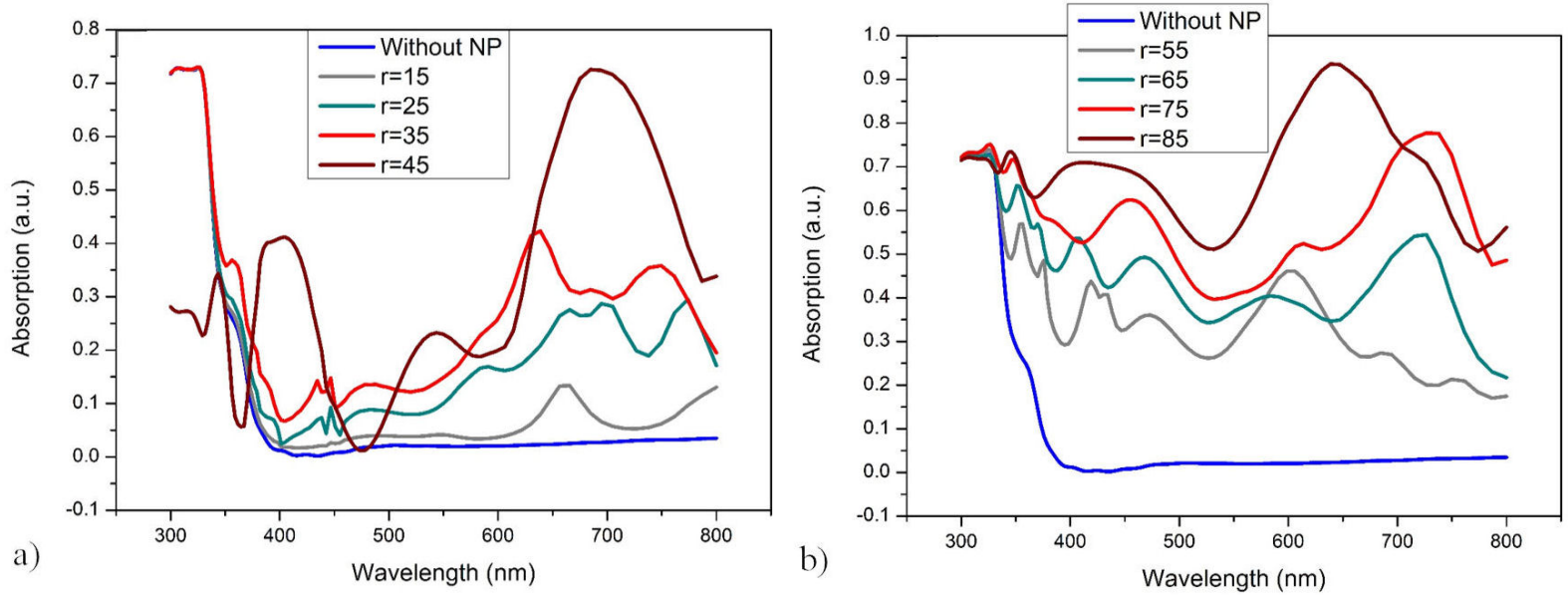

FIGURE 3. Absorption spectra of $\mathrm{TiO}_{2}$ layer with different size of Au nanoparticles.

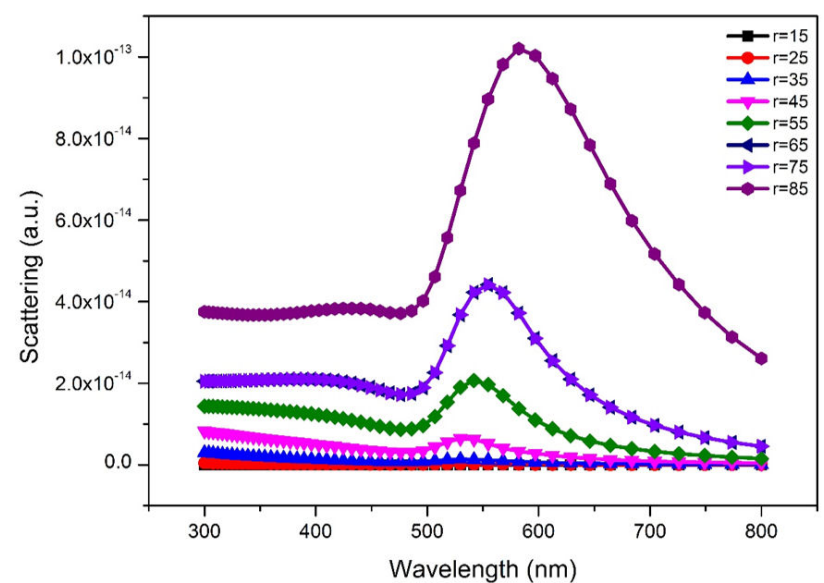

FIGURE 4. Scattering spectra of different size of Au nanoparticles.

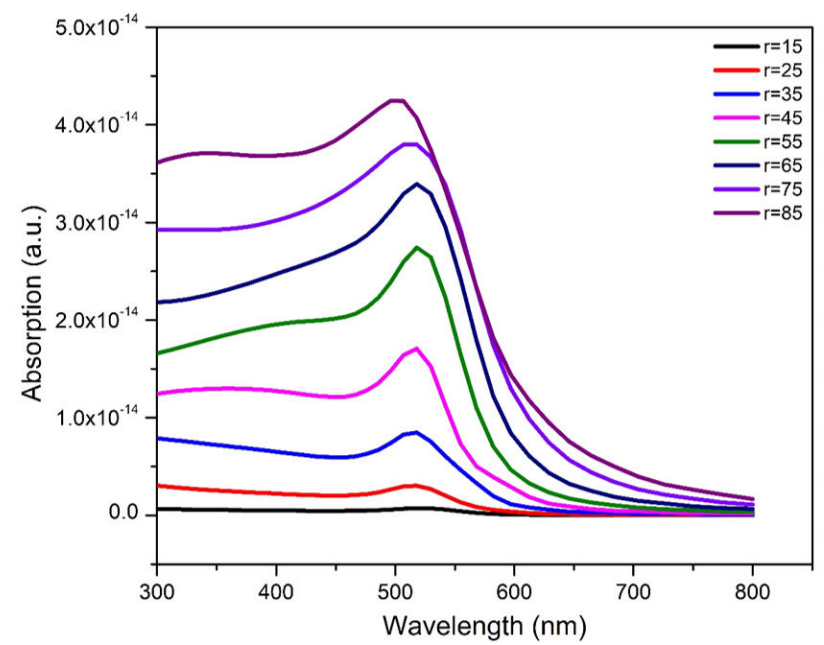

FIGURE 5. Absorption spectra of different size of Au nanoparticles.

vealed that the change in the size of the particles from $r=$ $85 \mathrm{~nm}$ to $r=15 \mathrm{~nm}$ gradually reduces the absorption spectrum.

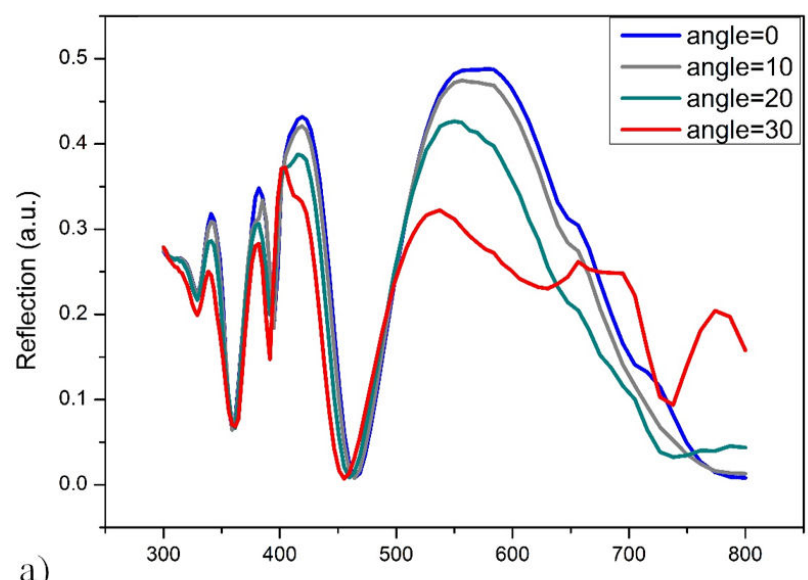

a)



FIGURE 6. Reflection of $\mathrm{Au} @ \mathrm{TiO}_{2}$ thin layer of non-zero injection angle source.

Bloch Boundary Conditions (BBCs) are similar to PBCs and are essentially required for a phase change across each period. The BBCs for a plane wave source were set at a nonzero injection angle. However, the zero-injection angle refers to the normal incidence of light on the layer of $\mathrm{TiO}_{2}$ thin film. 

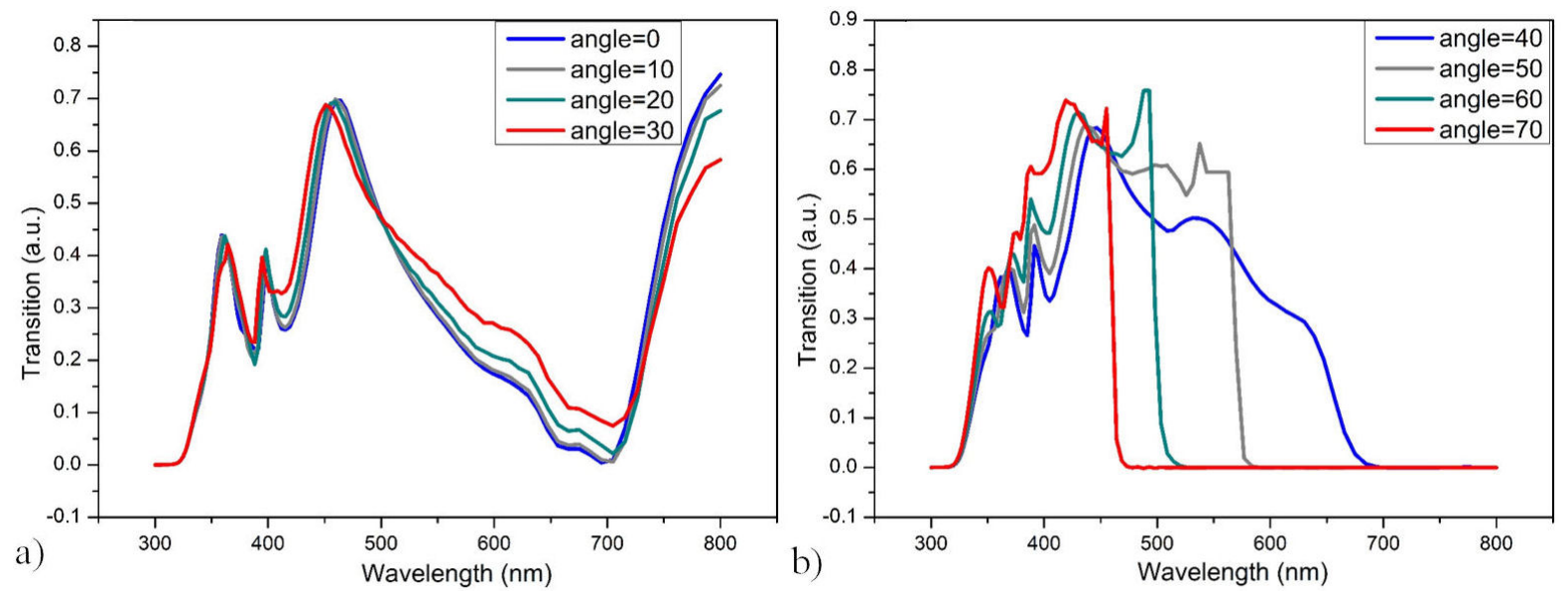

FIGURE 7. Transition of Au@ TiO2 thin layer of non-zero injection angle source.
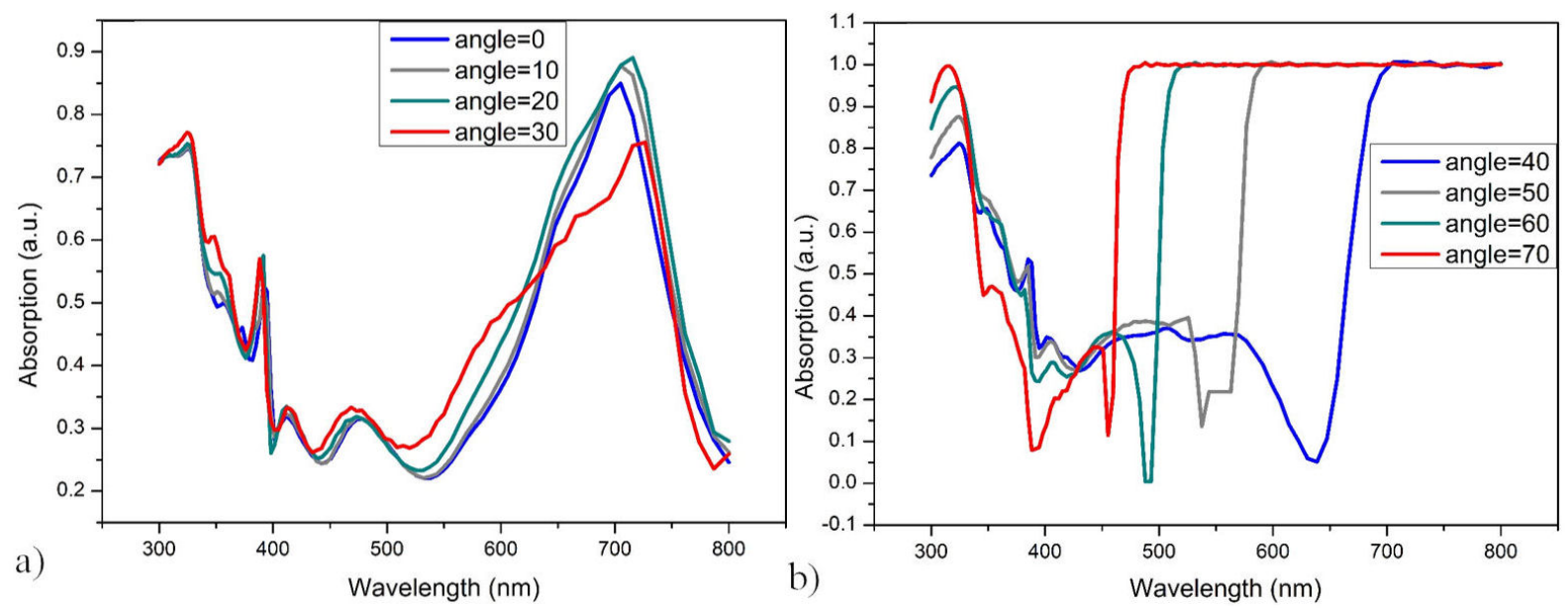

FIGURE 8. Absorption of $\mathrm{Au} @ \mathrm{TiO}_{2}$ thin layer of non-zero injection angle source.

To demonstrate the effect of a non-zero injection angle source on the improvement in absorption, a volume of the simulation was selected with a length of $0.1 \mu \mathrm{m}$, a width of $0.1 \mu \mathrm{m}$, and a height of $1 \mu \mathrm{m}$. It was evident that by increasing the plane wave angle injection source, light absorption significantly increased in the GNPs@ $\mathrm{TiO}_{2}$ thin film, as shown in Fig. 8. However, in the case of reflection of light, the changes were not considered in GNPs@ $\mathrm{TiO}_{2}$ thin-film except for the injection angle of $40^{\circ}$ that showed an increase, as can be seen in Fig. 6. On the contrary, it is clear from Fig. 7 that the decreasing light transition above $450 \mathrm{~nm}$ of wavelength with increasing in the angle of injection source. It is worth mentioning that at the light source injection angle of $70^{\circ}$ absorption reaches almost $100 \%$ between $450 \leq \lambda \leq 800 \mathrm{~nm}$ in GNPs@ $\mathrm{TiO}_{2}$ thin film. This implies that the best injection angle to achieve high performance of DSSC devices based on embedding GNPs on $\mathrm{TiO}_{2}$ thin film is $70^{\circ}$.

\section{Conclusion}

Increased optical absorption of a $\mathrm{TiO}_{2}$ semiconducting layer by embedding star-shaped GNPs in DSSCs was verified. A simulation was also performed to interpret the improvement of photocurrent by means of FDTD solution. The effects of the size of GNPs on light absorption and non-zero injection angle on the $\mathrm{TiO}_{2}$ layer were presented. The investigation of the size of GNPs has shown that the best optimum size for absorption is GNPs with a radius of $85 \mathrm{~nm}$, with an injection angle of $70^{\circ}$. This has revealed that these GNPs have multiple plasmon resonances resulting in polarization-dependent scattering with multiple spectral peaks, which correspond to the star-shaped structure's tips and branches. The quantum of light scattered and absorbed by the various sizes of GNPs, along with their effects, was also presented. This study indicates that the size of NPs in $\mathrm{TiO}_{2}$ thin film influences the level of light scattering and absorption. It is believed that DSSC with star-shaped GNPs embedded $\mathrm{TiO}_{2}$ layer records more PCE than traditional DSSC. Future research will focus 
on extending the model to a full DSSC package. Coupling of the optical model to an electrical charge transport model would enable a complete DSSC device optimization.

\section{Conflict of Interest}

The authors declare no conflict of interest.
1. D. Eli, P. Gyuk, M. Ahmad, G. Baba, and S. Sarki, Silver nanoparticles as artificial antennas for enhanced lightharvesting and charge transfer in dye-sensitized solar cells, Int. J. Mater. Sci. Appl. 5 (2016) 214-221, https://doi.org/ 10.11648/j.ijmsa 20160505.16.

2. D. Zhang, extitet al., Enhanced performance of dye-sensitized solar cells using gold nanoparticles modified fluorine tin oxide electrodes, J. Phys. D: Appl. Phys. 46 (2012) 024005, https : //doi.org/10.1088/0022-3727/46/2/024005.

3. T. Wan, S. Ramakrishna, and Y. Liu, Recent progress in electrospinning $\mathrm{TiO} 2$ nanostructured photo-anode of dye-sensitized solar cells, J. Appl. Polym. Sci. 135 (2018) 45649, https: //doi.org/10.1002/app.45649.

4. L. Zhang and Z.-S. Wang, Gold nanoparticles as an ultrathin scattering layer for efficient dye-sensitized solar cells, $J$. Mater. Chem. C 4 (2016) 3614-3620, https://doi .org/ 10.1039/C6TC00592F.

5. S. Sun, P. Song, J. Cui, and S. Liang, Amorphous TiO2 nanostructures: synthesis, fundamental properties and photocatalytic applications, Catal. Sci. Technol. 9 (2019) 4198-4215, https : //doi.org/10.1039/C9CY01020C.

6. R. Selvapriya, et al., Dual morphology titanium dioxide for dye sensitized solar cells, Ceram. Int. 45 (2019) 7268-7277, https://doi.org/10.1016/j.ceramint.2019. 01.008 .

7. N. Chander, et al., Size and concentration effects of gold nanoparticles on optical and electrical properties of plasmonic dye sensitized solar cells, Sol. Energy 109 (2014) 1123, https://doi.org/10.1016/j.solener.2014. 08.011 .

8. C. Andrei, et al., Plasmonic enhancement of dye sensitized solar cells via a tailored size-distribution of chemically functionalized gold nanoparticles, PLOS One 9 (2014) e109836, https://doi.org/10.1371/journal. pone.0109836.

9. J. Burschka, et al., Sequential deposition as a route to high-performance perovskite-sensitized solar cells, $\mathrm{Na}$ ture 499 (2013) 316-9, https://doi.org/10.1038/ nature12340.

10. Y. Yao, et al., Highly Efficient Sn-Pb Perovskite Solar Cell and High-Performance All-Perovskite Four-Terminal Tandem Solar Cell, Sol. RRL 3 (2019) 1900396, https: / / doi.org/10. 1002 /solr.201900396.

11. Q. Wang, et al., Enhanced performance of dye-sensitized solar cells by doping Au nanoparticles into photoanodes: a size effect study, J. Mater. Chem. A 1 (2013) 13524, https: //doi.org/10.1039/C3TA12692G.

12. K. W. Qadir, Z. Ahmad, K. Sulaiman, C. C. Yap, and F. Touati, Binary blend based dye sensitized photo sensor using PCPDTBT and MEH-PPV composite as a light sensitizer,
Synth. Met. 210 (2015) 392-397, https://doi.org/10. $1016 / j$.synthmet.2015.11.005.

13. A. Luque and S. Hegedus, Handbook of photovoltaic science and engineering. 2011: John Wiley \& Sons, https://doi. org/10.1002/0470014008.

14. P. Selvaraj, et al., Enhancing the efficiency of transparent dyesensitized solar cells using concentrated light, Sol. Energy Materl. Sol. Cells 175 (2018) 29-34, https://doi.org/ $10.1016 / j$.solmat.2017.10.006.

15. S. Saravanan, R. Kato, M. Balamurugan, S. Kaushik, and T. Soga, Efficiency improvement in dye sensitized solar cells by the plasmonic effect of green synthesized silver nanoparticles, J. Sci: Adv. Mater. Dev. 2 (2017) 418-424, https://doi. org/10.1016/j.jsamd.2017.10.004.

16. M. J. H. Pantho, N. A. Junnat, and M. J. Alam. Design and analysis the characteristics of a cost-effective polymer based bulk heterojunction tandem solar cell, in Proceedings of the 8th International Conference on Electrical and Computer Engineering, 2014, Dhaka, IEEE, https://doi.org/10.1109/ ICECE.2014.7026910.

17. F. Beck, A. Polman, and K. Catchpole, Tunable light trapping for solar cells using localized surface plasmons, J. Appl. Phys. 105 (2009) 114310, https://doi.org/10.1063/ 1.3140609 .

18. S. Mayumi, et al., Effect of Gold Nanoparticle Distribution in $\mathrm{TiO} 2$ on the Optical and Electrical Characteristics of Dye-Sensitized Solar Cells, Nanoscale Res. Lett. 12 (2017) 513, https://doi.org/10.1186/ s11671-017-2285-4.

19. B. O'Connor, K. H. An, K. P. Pipe, Y. Zhao, and M. Shtein, Enhanced optical field intensity distribution in organic photovoltaic devices using external coatings, Appl. Phys. Lett. 89 (2006) 233502, https : / / doi .org/10 .1063/1. 2399937.

20. M. Agrawal and P. Peumans, Broadband optical absorption enhancement through coherent light trapping in thin-film photovoltaic cells, Opt. Express 16 (2008) 5385-5396, https: //doi.org/10.1364/OE.16.005385.

21. D. H. Ko, et al., Photonic crystal geometry for organic solar cells, Nano Lett. 9 (2009) 2742-6, https : / / doi .org/10 . $1021 / \mathrm{n} 1901232 \mathrm{p}$.

22. J. R. Tumbleston, D. H. Ko, E. T. Samulski, and R. Lopez, Absorption and quasiguided mode analysis of organic solar cells with photonic crystal photoactive layers, Opt. Express 17 (2009) 7670-81, https://doi.org/10.1063/ 1.117513 .

23. H. J. Park, T. Xu, J. Y. Lee, A. Ledbetter, and L. J. Guo, Photonic color filters integrated with organic solar cells for energy harvesting, ACS Nano. 5 (2011) 7055-60, https://doi. org/10.1021/nn201767e. 
24. S. I. Na, et al., Efficient polymer solar cells with surface relief gratings fabricated by simple soft lithography, $A d v$. Funct. Mater. 18 (2008) 3956-3963, https://doi.org/ 10.1002 /adfm.200800683.

25. M. Niggemann, M. Glatthaar, A. Gombert, A. Hinsch, and V. Wittwer, Diffraction gratings and buried nano-electrodesarchitectures for organic solar cells, Thin Solid Films 451 (2004) 619-623, https://doi.org/10.1016/j.tsf. 2003.11 .028 .

26. D. H. Song, H. S. Kim, J. S. Suh, B. H. Jun, and W. Y. Rho, Multi-Shaped Ag Nanoparticles in the Plasmonic Layer of DyeSensitized Solar Cells for Increased Power Conversion Efficiency, Nanomaterials 7 (2017) 136, https://doi.org/ $10.3390 /$ nano7060136.

27. H. R. Stuart and D. G. Hall, Absorption enhancement in silicon-on-insulator waveguides using metal island films, Appl. Phys. Lett. 69 (1996) 2327-2329, https : //doi.org/10. $1063 / 1.117513$.

28. L. Yuan, F. Chen, C. Zheng, J. Liu, and N. Alemu, Parasitic absorption effect of metal nanoparticles in the dye-sensitized solar cells, Phys. Status Solidi A 209 (2012) 1376-1379, https : //doi.org/10.1002/pssa.201228099.

29. R. W. Qadir and K. W. Qadir, Shape Effects on Light Absorption and Scattering for Nanoparticles in Thin Organic Films, Eurasian J. Sci. Eng. 4 (2019) 49-54, http://eprints. tiu.edu.iq/id/eprint/199.

30. R. W. Qadir, Z. Ahmad, and K. Sulaiman, Effect of the shapes of nanostructures on the light absorption in organic thin films, J. Mod. Opt. 61 (2014) 636-640, https://doi.org/10. $1080 / 09500340.2014 .904020$.

31. R. W. Qadir, K. W. Qadir, and S. B. Aziz, Effect of Various Shapes of Silver Nanoparticles on the Performance of Plasmonic Solar Cells Active Layer, Zanco J. Pure Appl. Sci. 48-44 (2019) 31 https://doi.org/10.21271/zjpas.31. s4.7.
32. S. K. Cushing and N. Wu, Plasmon-enhanced solar energy harvesting, Electrochem Soc. Interface 22 (2013) 63-67, https : //doi.org/10.1149/2.F08132if.

33. H. Jung, et al., Enhanced photovoltaic properties and long-term stability in plasmonic dye-sensitized solar cells via noncorrosive redox mediator, ACS Appl. Mater. Interfaces 6 (2014) 19191-200, https : / doi .org/10 .1021/ am5051982.

34. G. P. Sahoo, et al., Synthesis and photo physical properties of star shaped gold nanoparticles, Colloids Surf. A 375 (2011) 30-34, https://doi.org/10.1016/j. colsurfa.2010.11.033.

35. C. L. Nehl, H. Liao, and J. H. Hafner, Optical properties of star-shaped gold nanoparticles, Nano Lett. 6 (2006) 683-8, https://doi.org/10.1021/n1052409y.

36. M. Gu, et al., Nanoplasmonics: a frontier of photovoltaic solar cells, Nanophotonics 1 (2012) 235-248, https://doi. org/10.1515/nanoph-2012-0180.

37. Z. Wang, U. Helmersson, and P.-O. Käll, Optical properties of anatase $\mathrm{TiO} 2$ thin films prepared by aqueous sol-gel process at low temperature, Thin Solid Films 405 (2002) 50-54, https: //doi.org/10.1016/S0040-6090(01)01767-9.

38. N.-F. Chiu, Y.-C. Tu, and T.-Y. Huang, Enhanced sensitivity of anti-symmetrically structured surface plasmon resonance sensors with zinc oxide intermediate layers, Sensors 14 (2014) 170-187, https://doi.org/10.3390/s140100170.

39. M. Shanmugam, M. F. Baroughi, and D. Galipeau, High VOC dye sensitised solar cell using RF-sputtered $\mathrm{TiO} 2$ compact layers, Electron. Lett. 45 (2009) 648-649, https : / / doi . org/ $10.1049 / \mathrm{el} .2009 .3527$.

40. L. Rodríguez-Lorenzo, et al., Zeptomol detection through controlled ultrasensitive surface-enhanced Raman scattering, $J$. Am. Chem. Soc. 131 (2009) 4616-4618, https://doi. org/10.1021/ja809418t. 